Pacific Journal of Mathematics

CHARACTERIZATION OF CROSSED PRODUCT (PROPERLY 


\title{
CHARACTERIZATION OF CROSSED PRODUCT (PROPERLY INFINITE CASE)
}

\author{
HIDEKI KOSAKI \\ Dedicated to the memory of Professor Henry Abel Dye
}

\begin{abstract}
We characterize a crossed product of a properly infinite factor by an outer action of a finite group.
\end{abstract}

0. Introduction. We will obtain a properly infinite version of characterization of crossed product algebras as a generalization of the recent result (explained below) in the $\mathrm{II}_{1}$-factor set-up.

In [6] Ocneanu announced classification of subfactors in the hyperfinite $\mathrm{II}_{1}$-factor. His work is built upon Jones' index theory [2] and deep analysis in the Pimsner-Popa paper [7], and this classification is based on a newly-introduced invariant called a paragroup. His announcement contains the following intrinsic characterization of crossed products: Let $M$ be a $\mathrm{II}_{1}$-factor with a subfactor $N,[M ; N]<\infty$. If $M \cap N^{\prime}=\mathrm{C} 1, M_{1} \cap N^{\prime}$ is abelian, and the depth of $M \supseteq N$ is 2 , then there exist a finite group $G$ and an outer action $\alpha$ of $G$ on $N$ such that $M=N \rtimes_{\alpha} G$. Here, $M_{1}$ is the basic extension of $M \supseteq N$, and the definition of a depth can be found in [6].

A proof of this beautiful characterization has not been written yet (although the result as well as its proof are implicit in [7]). The purpose of the present paper is to provide a complete proof of a properly infinite version of this characterization. Therefore, the notion of index explained in [4] has to be used here, and Ocneanu's conditions are replaced by the following (equivalent) conditions: $M \cap N^{\prime}$ $=\mathrm{C} 1, M_{1} \cap N^{\prime}$ is abelian, and $\operatorname{dim}_{\mathbf{C}}\left(M_{1} \cap N^{\prime}\right)=$ Index $E$. (In particular, Index $E$ is an integer.) Here, $E$ is a unique normal conditional expectation from $M$ onto $N$.

Our standard references on operator algebras are [8] and [9], and basic facts on index theory can be found in [2], [4], and [7]. Some auxiliary results will be proved in appendices. 
1. Notations and main theorem. Throughout let $M$ be a factor with a subfactor $N$, and let $E$ be a normal conditional expectation from $M$ onto $N$ satisfying Index $E<\infty$.

Let $H$ be the standard $L^{2}$-space of $M$ so that $M$ and $N$ are acting on $H$. Once a natural cone is fixed in $H$, one can perform the basic construction. As usual by $M_{1}$ we denote the basic extension of $M \supseteq N$, that is, $M_{1}=J N^{\prime} J=M \vee\{e\}^{\prime \prime}$, where $J$ is the modular conjugation on $H$ and $e$ is the projection arising from $E$. Let $E_{M}$ be the normal conditional expectation from $M_{1}$ onto $M$ constructed from $E^{-1} \cdot\left(E_{M}=(\operatorname{Index} E)^{-1} \times J E^{-1}(J \cdot J) J\right)$ (see Appendix I for details).

LEMMA 1. If $M_{1} \cap N^{\prime}$ is abelian, then $\operatorname{dim}_{C}\left(M_{1} \cap N^{\prime}\right) \leq$ Index $E$.

Proof. Let $\left\{p_{i}\right\}_{i=1,2, \ldots, m}$ be the minimal projections in $M_{1} \cap N^{\prime} . \mathrm{Re}-$ call that

$$
\operatorname{Index}\left(E \circ E_{M}\right)=(\operatorname{Index} E)\left(\operatorname{Index} E_{M}\right)=(\operatorname{Index} E)^{2} .
$$

Considering local indices $([4], 4)$, we estimate

$$
\begin{aligned}
(\operatorname{Index} E)^{2} & \geq \sum_{i=1}^{m} E \circ E_{M}\left(p_{i}\right)^{-1} \operatorname{Index}\left(E \circ E_{M}\right)_{p_{l}} \\
& \geq \sum_{i=1}^{m} E \circ E_{M}\left(p_{i}\right)^{-1} .
\end{aligned}
$$

Notice that $E \circ E_{M}\left(p_{i}\right)$ 's are positive scalars summing up to 1 . Therefore, the minimum of the above sum of the inverses is $m \times(1 / m)^{-1}=$ $m^{2}$ (and the minimum is attained only when all of $E \circ E_{M}\left(p_{i}\right)$ are exactly $1 / m)$.

Theorem 2. Assume that $M$ is properly infinite and Index $E=n \in$ $\mathbf{N}_{+}$. If $M \cap N^{\prime}=\mathbf{C} 1, M_{1} \cap N^{\prime}$ is abelian, and $\operatorname{dim}_{\mathbf{C}}\left(M_{1} \cap N^{\prime}\right)=n$, then there exist a finite group $G$ (of order $n$ ) and an outer action $\alpha$ of $G$ on $N$ such that $M$ is the crossed product $N \rtimes_{\alpha} G$. (Furthermore, $E$ is the canonical conditional expectation from $N \rtimes_{\alpha} G$ onto $N$.)

When $M=N \rtimes_{\alpha} G$ from the beginning, it is easy to check that all of the above conditions are indeed satisfied. It is an amusing exercise so that we leave it to the reader. We just remark that (i) $E$ in this case is the canonical expectation from $N \rtimes_{\alpha} G$ onto $N$, (ii) this exercise requires the characterization of an outer action obtained in [3]. 
2. Proof of Theorem. We still keep the assumptions at the beginning of $\S 1$. From now on, we will also assume that $M \cap N^{\prime}=\mathbf{C} 1$, Index $E=n \in \mathbf{N}_{+}$, and $M_{1} \cap N^{\prime}$ is abelian. (Hence, by Lemma 1, $\operatorname{dim}_{\mathbf{C}}\left(M_{1} \cap N^{\prime}\right) \leq n$.)

LemMA 3. Assume that $\operatorname{dim}_{\mathrm{C}}\left(M_{1} \cap N^{\prime}\right)=n$, and let $\left\{p_{i}\right\}_{i=1,2, \ldots, n}$ be the minimal projections in $M_{1} \cap N^{\prime}$. Then we have $E_{M}\left(p_{i}\right)=(1 / n) 1$ for each $i$. In particular, we get $\left\{p_{1}, p_{2}, \ldots, p_{n}\right\}=\left\{p \in\left(M_{1} \cap N^{\prime}\right)_{\text {proj }}\right.$; $\left.E_{M}(p)=1 / n\right\}$.

Proof. Under the present assumptions, the inequalities in the proof of Lemma 1 read:

$$
\begin{aligned}
n^{2} & \geq \sum_{i=1}^{n} E \circ E_{M}\left(p_{i}\right)^{-1} \operatorname{Index}\left(E \circ E_{M}\right)_{p_{l}} \\
& \geq \sum_{i=1}^{n} E \circ E_{M}\left(p_{i}\right)^{-1} \geq n \times\left(\frac{1}{n}\right)^{-1}=n^{2} .
\end{aligned}
$$

We thus conclude that $E \circ E_{M}\left(p_{i}\right)=1 / n$ (and $\operatorname{Index}\left(E \circ E_{M}\right)_{p_{t}}=1$ ). Since $M \cap N^{\prime}=\mathrm{C} 1, E_{M}\left(p_{i}\right)$ is already a scalar and we get

$$
1 / n=E \circ E_{M}\left(p_{i}\right)=E_{M}\left(p_{i}\right)
$$

We next investigate a "Weyl group" as in [7]. The minimal projections $\left\{p_{i}\right\}_{i=1,2, \ldots, n}$ will be fixed from now on. We may and do assume that $p_{1}=e$. For each $p_{i}$, we choose a partial isometry $v_{i} \in M_{1}$ satisfying $v_{i} v_{i}^{*}=p_{i}$ and $v_{i}^{*} v_{i}=e$. (It is always possible if $M$ is of type III. It is also possible if $M$ is of type either $\mathrm{II}_{1}$ or $\mathrm{II}_{\infty}$. In fact, in these cases, $E$ comes from the unique trace and Lemma 3 guarantees that $p_{i}$ and $e$ have the same trace value. See Appendix II.) By Lemma 3.3, [4], $u_{i}=n E_{M}\left(v_{i} e\right) \in M$ satisfies $v_{i}=v_{i} e=u_{i} e$. We thus get

$$
\left\{\begin{array}{l}
p_{i}=u_{i} e u_{i}^{*}, \\
e=e u_{i}^{*} u_{i} e=E\left(u_{i}^{*} u_{i}\right) e, \text { that is, } E\left(u_{i}^{*} u_{i}\right)
\end{array}\right.
$$

Applying $E_{M}$ to (1), from Lemma 3 we get

$$
\begin{aligned}
1 / n & =E_{M}\left(p_{i}\right)=E_{M}\left(u_{i} e u_{i}^{*}\right)=u_{i} E_{M}(e) u_{i}^{*} \quad\left(\text { since } u_{i} \in M\right) \\
& =(1 / n) u_{i} u_{i}^{*} .
\end{aligned}
$$

Hence $u_{i}$ is a coisometry. Then (2) means that the operator $1-u_{i}^{*} u_{i} \geq$ 0 satisfies $E\left(1-u_{i}^{*} u_{i}\right)=0$. The expectation $E$ being faithful, we have 
$1-u_{i}^{*} u_{i}=0$, and consequently $u_{i}$ is a unitary in $M$. Since $v_{i}^{*} v_{j}=0$ $(i \neq j)$, we compute

$$
0=e u_{i}^{*} u_{j} e=E\left(u_{i}^{*} u_{j}\right) e, \text { that is, } E\left(u_{i}^{*} u_{j}\right)=0 .
$$

Let $\mathcal{N}(N)$ be the set of all unitaries $u$ in $M$ satisfying $u N u^{*}=N$. Notice that the $n$ unitaries $u_{1}, u_{2}, \ldots, u_{n}$ belong to $\mathscr{N}(N)$. In fact, for each $y \in N$, we compute

$$
y u_{i} e u_{i}^{*}=y p_{i}=p_{i} y=u_{i} e u_{i}^{*} y
$$

because of $p_{i} \in M_{1} \cap N^{\prime}$. But this means that $u_{i}^{*} y u_{i} \in M$ commutes with $e$, that is, $u_{i}^{*} y u_{i} \in N$. Obviously $\mathcal{N}(N)$ forms a group (under the ordinary operator multiplication) and the unitary group $\mathscr{U}(N)$ is a normal subgroup in $\mathscr{N}(N)$.

Definition 4. The quotient group $\mathscr{N}(N) / \mathscr{U}(N)$ is denoted by $G$ (the Weyl group of $M \supseteq N$ ).

For each $u \in \mathscr{N}(N), u e u^{*}$ is a projection in $M_{1} \cap N^{\prime}$. In fact, for each $y \in N$, we compute

$$
\begin{aligned}
u_{e} u^{*} y & =u e\left(u^{*} y u\right) u^{*}=u\left(u^{*} y u\right) e u^{*} \quad\left(\text { since } u^{*} y u \in N\right) \\
& =y e u^{*} .
\end{aligned}
$$

We also notice that

$$
E_{M}\left(u e u^{*}\right)=u E_{M}(e) u^{*}=(1 / n) u u^{*}=1 / n .
$$

Therefore, $u e u^{*}$ has to be one of $p_{i}$ 's (see Lemma 3).

LEMMA 5 (Proposition 1.7, [7]). The Weyl group $G$ is a finite group of order $n$. More precisely, $u \in \mathscr{N}(N) \rightarrow u^{*} \in\left\{p \in\left(M_{1} \cap N^{\prime}\right)_{\text {proj }}\right.$; $\left.E_{M}(p)=1 / n\right\}$ induces the bijection:

$$
[u] \in G \rightarrow u e u^{*} \in\left\{p \in\left(M_{1} \cap N^{\prime}\right)_{\mathrm{proj}} ; E_{M}(p)=1 / n\right\} .
$$

Proof. Since $e$ commutes with an arbitrary element in $N$, the second map is certainly well-defined. To show the injectivity, let us assume $u e u^{*}=\tilde{u} e \tilde{u}^{*}(u, \tilde{u} \in \mathcal{N}(N))$. Then the unitary $\tilde{u}^{*} u \in M$ commutes with $e$, that is, $\tilde{u}^{*} u \in N$ and $[\tilde{u}]=[u]$. The surjectivity follows from $p_{i}=u_{i} e u_{i}^{*}$.

We have shown that the Weyl group $G$ (a finite group of order $n$ ) is parametrized by:

$$
\left\{p \in\left(M_{1} \cap N^{\prime}\right)_{\text {proj }} ; E_{M}(p)=1 / n\right\}=\left\{p_{1}, p_{2}, \ldots, p_{n}\right\} .
$$


Therefore, in what follows, we shall write

$$
\begin{gathered}
\left\{p \in\left(M_{1} \cap N^{\prime}\right)_{\text {proj }} ; E_{M}(p)=1 / n\right\}=\left\{p_{g}\right\}_{g \in G} \\
\left(p_{1}=e, \text { where } 1 \text { denotes the unit in } G\right)
\end{gathered}
$$

and

$$
g=\left[u_{g}\right] \in G=\mathscr{N}(N) / \mathscr{U}(N) \quad\left(\text { with } u_{1}=1\right) .
$$

Notice that $\operatorname{Ad} u_{g}(g \neq 1)$ is an outer automorphism of $N$. In the above argument, a choice of a partial isometry $v_{i}$ (hence that of a unitary $u_{g}$ ) is highly non-unique. Can we choose $n$ representative unitaries $u_{g}, g \in G$, in such a way that $u_{g}$ 's themselves form a group (in $\mathscr{N}(N))$ ? This is a cohomological question. Indeed, since [ $u_{g}$ ]'s form a group, we obtain a 2-cocycle on $G$ (whose values are in the unitary group $\mathscr{U}(N)$ ). The group $G$ being finite, it is known that a 2-cocycle is a coboundary. (This is due to Sutherland. See [8] for example.) This means that by perturbing $u_{g}$ by a unitary in $N$ we can assume that $u_{g}$ 's form a group. Notice that this perturbation does not destroy the property $u_{g} e u_{g}^{*}=p_{g}$ (since $e$ and $p_{g}$ belong to $M_{1} \cap N^{\prime}$ ). By setting $\alpha_{g}=\left.\operatorname{Ad} u_{g}\right|_{N}$ (with the perturbed $u_{g}$ 's), we obtain an outer action $\alpha$ on $N$.

Proof of Theorem 2. For any $x$ in $M$, we compute

$$
x e=1 x e=\sum_{g \in G} u_{g} e u_{g}^{*} x e=\sum_{g \in G} u_{g} E\left(u_{g}^{*} x\right) e .
$$

Hence $x=\sum_{g \in G} u_{g} x_{g}$ with $x_{g}=E\left(u_{g}^{*} x\right) \in N$. Also the "coefficients" $x_{g}$ 's are uniquely determined by $x$ since $E\left(u_{h}^{*} u_{g}\right)=0, g \neq h$, obviously remains valid after the above-mentioned perturbation. (This is an " $N$-module decomposition" of $M$ obtained in [7].) Let $N \rtimes_{\alpha} G$ be the crossed product generated by the usual generators $\pi_{\alpha}(x)(x \in N)$ and $\lambda_{g}(g \in G)$. It is easy to see that $\sum_{g \in G} u_{g} x_{g} \rightarrow \sum_{g \in G} \lambda_{g} \pi_{\alpha}\left(x_{g}\right)$ gives rise to an isomorphism from $M$ onto $N \rtimes_{\alpha} G$. Since $E\left(u_{g}^{*} y\right)=0$ $(g \neq 1, y \in N)$ and $E\left(u_{1}^{*} y\right)=y(y \in N)$, a generic element $x=$ $\sum_{g \in G} u_{g} x_{g} \in M$ belongs to the subfactor $N$ if and only if $x_{g}=0$ for all $g \neq 1$. Thus the above-mentioned isomorphism from $M$ onto $N \rtimes_{\alpha} G$ sends $N$ onto $\pi_{\alpha}(N)$, that is, $N \subseteq M$ and $\pi_{\alpha}(N) \subseteq N \rtimes_{\alpha} G$ are conjugate.

REMARK 6. By "dualizing" a situation, we can prove the following characterization of fixed point subalgebras: Assume Index $E=n \in N$. If $M \cap N^{\prime}=\mathrm{C} 1, M_{2} \cap M^{\prime}$ is abelian, and $\operatorname{dim}_{\mathbf{C}}\left(M_{2} \cap M^{\prime}\right)=n$, then 
there exists an outer action of a finite group $G$ (of order $n$ ) on $M$ such that $N=M_{G}$, the fixed point subalgebra. Here, $M_{2}$ denotes the basic extension of $M_{1} \supseteq M$. (See [6] for the $\mathrm{II}_{1}$-factor case.)

This result can be proved based on the general theory on actions and coactions of a group (and a "group dual"). However, we will briefly describe a direct proof in what follows.

Our previous arguments guarantee existence of $n$ unitaries $\left\{u_{g}\right\}_{g \in G}$ in $M_{1}$ such that $u_{g} M u_{g}^{*}=M$ and $u_{g}$ 's form a group (in $\mathscr{U}\left(M_{1}\right)$ ) of order $n$. Let $J_{1}$ be the modular conjugation on $L^{2}\left(M_{1}\right)$. Then $v_{g}=J_{1} u_{g} J_{1}$ normalizes $M_{2}=J_{1} M^{\prime} J_{1}$ so that we get an action of $G$ on $M_{2}$. This is an outer action and we have $M_{2} \supseteq\left(M_{2}\right)_{G} \supseteq M_{1}$. Thanks to [1], the index of the (unique) expectation from $M_{2}$ onto $\left(M_{2}\right)_{G}$ is $n$ so that we conclude

$$
\left(M_{2}\right)_{G}=M_{1} \text {. }
$$

Since $M$ and $N$ are properly infinite, they have a common cyclic and separating vector $\zeta$ in $H=L^{2}(M)$. Let $J_{M}, J_{N}$ be the modular conjugation arising from $(M, \zeta)$ and $(N, \zeta)$ respectively. Let $\gamma=$ Ad $J_{N} J_{M}$ be Longo's canonical endomorphism from $M$ to $N$, [5]. It is easy to see that $N \supseteq \gamma(M) \supseteq \gamma(N)$ are downward basic extensions. Also notice that $M_{1}=J_{M} N^{\prime} J_{M}=J_{M} J_{N} N J_{N} J_{M}$ (acting on $H$ with a cyclic and separating vector $\zeta$ ). The modular conjugation $J_{1}$ coming from $\left(M_{1}, H, \zeta\right)$ is $\left(J_{M} J_{N}\right) J_{N}\left(J_{M} J_{N}\right)^{*}=J_{M} J_{N} J_{M}$, so that $M_{2}=J_{1} M^{\prime} J_{1}=J_{M} J_{N} M J_{N} J_{M}$. (All the upward and downward basic extensions can be constructed by using the single Hilbert space H.) Notice that $\gamma(M) \cap \gamma(N)^{\prime}=\gamma\left(M \cap N^{\prime}\right)=\gamma(\mathbf{C} 1)=\mathbf{C} 1$. We also compute

$$
\begin{aligned}
M \cap \gamma(M)^{\prime} & =M \cap\left(J_{N} J_{M} M J_{M} J_{N}\right)^{\prime}=M \cap\left(J_{N} J_{M} M^{\prime} J_{M} J_{N}\right) \\
& =J_{N} J_{M}\left\{\left(J_{M} J_{N} M J_{N} J_{M}\right) \cap M^{\prime}\right\} J_{M} J_{N} \\
& =J_{N} J_{M}\left(M_{2} \cap M^{\prime}\right) J_{M} J_{N} .
\end{aligned}
$$

So the inclusion $\gamma(M) \supseteq \gamma(N)$ satisfies the same assumptions as $M \supseteq$ $N$. Thus, by applying (3) to the inclusion $\gamma(M) \supseteq \gamma(N)$, we obtain the result.

APPENDIX I. Natural cone and basic construction.

As mentioned in the main body of the paper, a basic construction is determined after a natural cone is fixed. Although this is a folklore for specialists, this was not explicitly explained in [4]. Thus to minimize confusion we will make this point clear in the appendix. 
Let $E: M \rightarrow N$ be as the beginning of $\S 1$ and let $H$ be a Hilbert space on which $M$ is acting standardly. We now fix a natural cone $\mathrm{H}^{+}$ $\left(\subseteq H\right.$ ) and the associated modular conjugation $J$. The commutant $N^{\prime}$ is independent of the choice of $\mathrm{H}^{+}$and $J$ is solely determined by $\mathrm{H}^{+}$ so that obviously $M_{1}=J N^{\prime} J$ is determined by $H^{+}$. Actually we have more. Namely the involved projection $e=e_{N}\left(M_{1}=\langle M, e\rangle\right)$ is also uniquely determined by $H^{+}$(although $e$ was defined after choosing a faithful normal state on $N,[4])$.

LEMMA A. Let $\phi_{i}(i=1,2)$ be faithful normal states on $N$ with $\phi_{i} \circ E=\omega_{\xi_{l}}\left(\xi_{i} \in H^{+}\right)$. Define the projections $e_{i}(i=1,2)$ by $e_{i} x \xi_{i}=$ $E(x) \xi_{i}(x \in M)$. Then the two projections $e_{i}$ in $N^{\prime}$ are identical.

Proof. For $x \in M$ and $t \in \mathbf{R}$, we compute

$$
\begin{aligned}
& e_{1} x\left(D\left(\phi_{2} \circ E\right) ; D\left(\phi_{1} \circ E\right)\right)_{t} \xi_{1}=E(x)\left(D\left(\phi_{2} \circ E\right) ; D\left(\phi_{1} \circ E\right)\right)_{t} \xi_{1} \\
& \quad=E(x)\left(D\left(\phi_{2} \circ E\right) ; D\left(\phi_{1} \circ E\right)\right)_{t} \xi_{1}
\end{aligned}
$$

because of $\left(D\left(\phi_{2} \circ E\right) ; D\left(\phi_{1} \circ E\right)\right)_{t}=\left(D \phi_{2} ; D \phi_{1}\right)_{t} \in N$. The two $H$ valued functions

$$
\left\{\begin{array}{l}
f(t)=e_{1} x\left(D\left(\phi_{2} \circ E\right) ; D\left(\phi_{1} \circ E\right)\right)_{t} \xi_{1}, \\
g(t)=E(x)\left(D\left(\phi_{2} \circ E\right) ; D\left(\phi_{1} \circ E\right)\right)_{t} \xi_{1}
\end{array}\right.
$$

extend to bounded continuous functions on $-1 / 2 \leq \operatorname{Im} z \leq 0$ which are analytic in the interior. The previous computation means $f(t)=$ $g(t), t \in \mathbf{R}$. Using the uniqueness of analytic continuation, we get $f(z)=g(z)$ and in particular $(z=-i / 2)$

$$
e_{1}\left(x \xi_{2}\right)=E(x) \xi_{2}
$$

which means $e_{1}=e_{2}$.

Next we investigate the effect coming from a different natural cone (possibly in a different Hilbert space). So let us assume that $M$ is also acting standardly on a Hilbert space $K$ and that $K^{+}(\subseteq K)$ is a natural cone with the associated modular conjugation $\tilde{J}$. Let $\phi$ be a faithful normal state on $N$ with $\phi \circ E(x)=(x \xi, \xi)_{H}=(x \zeta, \zeta)_{K}\left(\xi \in H^{+}\right.$, $\left.\zeta \in K^{+}\right)$. Consider the usual projections $e$ and $\tilde{e}$ given by

$$
\text { ex } \xi=E(x) \xi, \quad \tilde{e} x \zeta=E(x) \zeta \quad(x \in M) .
$$

( $e$ is acting on $H$ while $\tilde{e}$ is acting on $K$.) A linear map $x \zeta \in M \zeta$ $(\subseteq K) \rightarrow x \xi \in M \xi(\subseteq H)$ extends to a surjective isometry $u$ from $K$ onto $H$. For each $x \in M$ we compute

$$
u \tilde{e} u^{*} x \xi=u \tilde{e} x \zeta=u E(x) \zeta=E(x) \xi,
$$


which means $u \tilde{e} u^{*}=e$. It is also easy to see $u x u^{*}=x(x \in M)$. Here, the right (resp. left) side $x$ is understood to be acting on $K$ (resp. $H$ ). Therefore, $\rho=\operatorname{Ad}(u): B(K) \rightarrow B(H)$ sends $\langle M, \tilde{e}\rangle$ onto $\langle M, e\rangle$ in such a way that

$$
\left\{\begin{array}{l}
\rho(x)=x \quad(x \in M), \\
\rho(\tilde{e})=e .
\end{array}\right.
$$

Consequently basic extensions coming from different natural cones can be identified. We recall that $J$ and $\tilde{J}$ are also related via $u$. It is well known (and can be proved easily) that $u \tilde{J} u^{*}=J$. "Basic extensions of $E$ " are "conjugate" via the above $\rho$. This follows from the definition of $E_{M}$ (involving $E^{-1}$ and a $J$-operator) and the above comparison of two $J$-operators. But we can also argue in the following way. Namely, the "basic extension" $E_{M}$ is characterized as a unique normal conditional expectation satisfying $E_{M}(e)=1$. Therefore, $E_{M}:\langle M, e\rangle \rightarrow M$ and $\left(E_{M}\right)^{\sim}:\langle M, \tilde{e}\rangle \rightarrow M$ are related by

$$
\left(E_{M}\right)^{\sim}(x)=E_{M}(\rho(x)) .
$$

APPENDIX II. Type $\mathrm{II}_{\infty}$ case.

Let $E: M \rightarrow N$ be as before with Index $E<\infty$. In this appendix we will show that $E$ comes from the unique trace on $M$ if $M \cap N^{\prime}=\mathrm{C} 1$ and $M$ (or equivalently $N$ ) is of type $\mathrm{II}_{\infty}$. Let $\operatorname{tr}$ (resp. $\tau$ ) be the unique (up to a scalar) faithful semi-finite normal trace on $M$ (resp. $N)$. A real catch here is to show the semi-finiteness of $\operatorname{tr}$ on $N$.

LEMMA B. Under the above assumptions, we have $\tau \circ E=\lambda \operatorname{tr}$ for some $\lambda>0$. (Hence, $\operatorname{tr}$ is semi-finite on $N$ and $E$ comes from tr.)

Proof. Since $\tau \circ E$ is semi-finite (on $M$ ), there exists a unique positive self-adjoint operator $h$ (the Radon-Nikodym derivative relative to $\mathrm{tr}$ ) affiliated with $M$ such that $\tau \circ E=\operatorname{tr}(h \cdot)$. For each $x \in N$ and $t \in \mathbf{R}$, we compute

$$
\begin{aligned}
h^{i t} x h^{-i t} & =\sigma_{t}^{\tau \circ E}(x) \quad(\text { since } h \text { is the Radon-Nikodym derivative) } \\
& =\sigma_{t}^{\tau}(x) \quad(\text { since } x \text { is in } N) \\
& =x .
\end{aligned}
$$

This means that $h$ commutes strongly with an arbitrary element in $N$. The relative commutant $M \cap N^{\prime}$ being trivial, we must have $h=\lambda 1$ for some $\lambda>0$ and $\tau \circ E=\lambda \mathrm{tr}$. 
Acknowledgment. The author is grateful to the referee for suggesting some simplification of the arguments in the previous version of the article.

\section{REFERENCES}

[1] P. L. Aubert, Théorie de Galois pour une $W^{*}$-algèbre, Comment. Math. Helvetici, 39 (1976), 411-433.

[2] V. Jones, Index for subfactors, Invent. Math., 72 (1983), 1-25.

[3] R. Kallman, A generalization of free action, Duke Math. J., 36 (1969), 781-789.

[4] H. Kosaki, Extension of Jones' theory on index to arbitrary factors, J. Funct. Anal., 66 (1986), 123-140.

[5] R. Longo, Simple injective factors, Adv. in Math., 63 (1987), 152-171.

[6] A. Ocneanu, Galois theory for operator algebras, a talk given at Univ. California, Santa Barbara, (1986).

[7] M. Pimsner and S. Popa, Entropy and index for subfactors, Ann. Sci. École Norm. Sup, 19 (1986), 57-106.

[8] S. Stratila, Modular Theory of Operator Algebras, Abacuss Press, Tunbridge Wells, 1981.

[9] M. Takesaki, Theory of Operator Algebras I, Springer Verlag, New York, 1979.

Received February 25, 1988.

College of General Education

KYUSHU UNIVERSITY

FUKUOKA, 810, JAPAN 



\section{PACIFIC JOURNAL OF MATHEMATICS EDITORS}

\author{
V. S. VARADARAJAN \\ (Managing Editor) \\ University of California \\ Los Angeles, CA 90024 \\ Herbert Clemens \\ University of Utah \\ Salt Lake City, UT 84112 \\ THOMAS ENRIGHT \\ University of California, San Diego \\ La Jolla, CA 92093
}

R. FINN

Stanford University

Stanford, CA 94305

HeRmanN FlaschKa

University of Arizona

Tucson, AZ 85721

VAUGHAN F. R. JONES

University of California

Berkeley, CA 94720

STEVEN KeRCKHOFF

Stanford University

Stanford, CA 94305
RoBION KIRBY

University of California

Berkeley, CA 94720

C. C. MOore

University of California

Berkeley, CA 94720

HAROLD STARK

University of California, San La Jolla, CA 92093

\section{ASSOCIATE EDITORS}
R. ARENS
E. F. BECKENBACH
B. H. NeUmanN
F. WolF
K. YosHIDA (1906-1982)

\section{SUPPORTING INSTITUTIONS}
UNIVERSITY OF ARIZONA
UNIVERSITY OF BRITISH COLUMBIA CALIFORNIA INSTITUTE OF TECHNOLOGY UNIVERSITY OF CALIFORNIA MONTANA STATE UNIVERSITY UNIVERSITY OF NEVADA, RENO NEW MEXICO STATE UNIVERSITY OREGON STATE UNIVERSITY
UNIVERSITY OF OREGON UNIVERSITY OF SOUTHERN CALIFORNIA STANFORD UNIVERSITY UNIVERSITY OF HAWAII UNIVERSITY OF TOKYO UNIVERSITY OF UTAH WASHINGTON STATE UNIVERSITY UNIVERSITY OF WASHINGTON 


\section{Pacific Journal of Mathematics}

\section{Vol. 137, No. $1 \quad$ January, 1989}

V. S. Varadarajan, Henry Abel Dye $\ldots \ldots \ldots \ldots \ldots \ldots \ldots \ldots \ldots \ldots$ iii

Huzihiro Araki, An application of Dye's theorem on projection lattices to orthogonally decomposable isomorphisms $\ldots \ldots \ldots \ldots \ldots \ldots \ldots \ldots \ldots$

Richard Arens, The limit of a sequence of squares in an algebra need not be

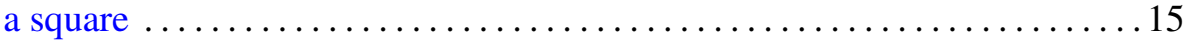

William Arveson, An addition formula for the index of semigroups of

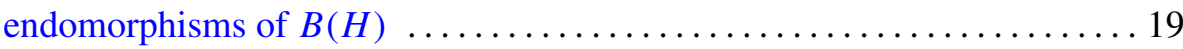

Robert James Blattner and Susan Montgomery, Crossed products and

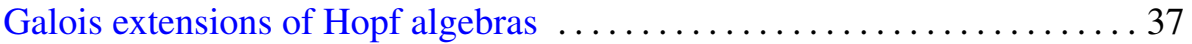

Erik Christensen and Allan M. Sinclair, On the vanishing of $H^{n}\left(\mathscr{A}, \mathscr{A}^{*}\right)$

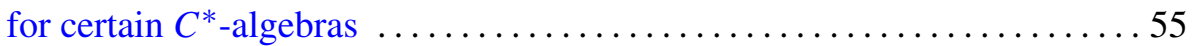

Philip C. Curtis, Jr. and Michael M. Neumann, Nonanalytic functional

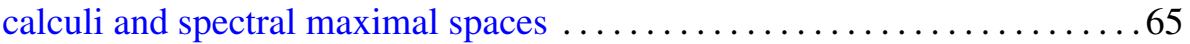

George A. Elliott and David E. Handelman, Addition of $C^{*}$-algebra extensions

Yaakov Friedman and Bernard Russo, Some affine geometric aspects of

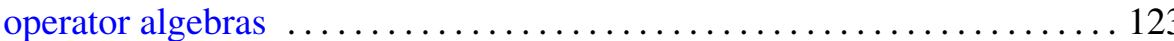

Valentin Ya. Golodets and Sergey D. Sinelshchikov, Regularization of actions of groups and groupoids on measured equivalence relations . . . 145

Irving Kaplansky, CCR-rings 155

Hideki Kosaki, Characterization of crossed product (properly infinite case)

Gert Kjærgaard Pedersen, Three quavers on unitary elements in $C^{*}$-algebras

Sorin Popa, Relative dimension, towers of projections and commuting squares of subfactors

Martin E. Walter, On a new method for defining the norm of

Fourier-Stieltjes algebras 